\title{
Synergistic Effect of Baicalin and Adriamycin in Resistant HL-60/ADM Leukaemia Cells
}

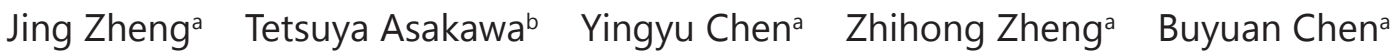 \\ Minhui Lin ${ }^{\mathrm{a}}$ Tingbo Liu ${ }^{\mathrm{a}}$ Jianda $\mathrm{Hu}^{\mathrm{a}}$
}

aFujian Institute of Hematology, Fujian Provincial Key Laboratory on Hematology, Fujian Medical University Union Hospital, Fuzhou, China; 'bepartment of Neurosurgery, Hamamatsu University School of Medicine, Handayama, Hamamatsu-city, Shizuoka, Japan

\section{Key Words}

Baicalin • Adriamycin • Leukaemic HL-60/ADM cells • Multidrug resistance • PI3K/Akt signalling pathway

\begin{abstract}
Background/Aims: The present study was designed to investigate the expression of multidrug resistance (MDR)-related genes, verify the synergistic effects of baicalin and Adriamycin (ADM) and investigate the related mechanisms in ADM-resistant leukaemic HL-60/ADM cells. Methods: We used a HL-60/ADM cell line. Cytotoxicity and flow cytometry assays were employed to verify the cytotoxic effects of baicalin. Real-time polymerase chain reaction and Western blotting assays were used to assess the expression of MDR-related genes and the changes in gene expression (both MDR-related and PI3K/Akt pathway-related) induced by administration of baicalin. Results: We found that only multidrug resistance protein 1 (MRP1), lung resistance-related protein (LRP) and $\mathrm{BCl}-2$ genes were expressed in both $\mathrm{HL}-60$ and HL-60/ADM cells. HL-60/ADM cells exhibited significantly higher expression $(p<0.05)$. We also observed that low-dose baicalin ( 5 and $10 \mu \mathrm{mol} / \mathrm{L}$ ) can induce growth inhibition and apoptotic effects on HL-60/ADM cells by increasing the intracellular accumulation of ADM. The synergistic effect of baicalin and ADM was verified. Concerning the potential mechanisms involved in this process, we showed that baicalin down-regulated the expression of several MDR-related and PI3K/Akt pathway-related genes. Conclusions: We confirmed the increased expression of MRP1, LRP and $\mathrm{Bcl}-2$ genes in HL-60/ADM cells compared to regular HL-60 cells, which are recommended for future investigation on MDR. The present study provided evidence of the synergistic effect of baicalin and ADM in HL-60/ADM cells. Therefore, baicalin may be considered as a potential therapeutic agent against resistant leukaemia. Suppression of the PI3K/Akt signalling pathway, followed by inhibition of the expression of MDR-related genes may be a common mechanism in combination treatments with ADM for the reduction of resistance to ADM.




\section{Cellular Physiology Cell Physiol Biochem 2017;43:419-430 \begin{tabular}{ll|l} 
and Biochemistry Published online: September 01, 2017 & $\begin{array}{l}\text { (c) } 2017 \text { The Author(s). Published by S. Karger AG, Basel } \\
\text { www.karger.com/cpb }\end{array}$ \\
\hline
\end{tabular} \\ Zheng et al.: Synergistic Effect of Baicalin and Adriamycin in HL-60/ADM Cells}

\section{Introduction}

Multidrug resistance (MDR) is one of the major causes of chemotherapy failure in the treatment of leukaemia and disease relapse. Adriamycin (ADM) is an important chemotherapy agent, usually combined with other therapeutic agents to treat leukaemia. In advanced or recurrent patients, resistance to ADM may gradually develop and greatly reduce treatment efficacy. Reversing resistance by recovering sensitivity of leukaemic cells to ADM is an urgent challenge for clinicians. The use of classic MDR modifiers is not satisfactory due to their high toxicity. Newer strategies to reverse MDR in the treatment of acute leukaemia are currently being investigated, with traditional herbs receiving considerable attention.

Baicalin is a type of flavonoid extracted from the Chinese medicinal herb Scutellaria baicalensis Georgi (Huang Qin) widely used in various disease models [1-3]. It has been documented that baicalin exerts effects on the inhibition of cancer cells via apoptosis and cell-cycle arrest $[4,5]$ in many types of tumours such as prostate cancer [4, 6], Burkitt lymphoma [7], oesophageal squamous cell carcinoma [8], etc. With regard to haematological malignancies, many studies investigated these anticancer effects. Lu et al. indicated that baicalin-induced apoptosis is conducted via expression of the Gadd153 and mitochondrialdependent pathway [9]. A more recent study found that activation of caspase- 3 may play a role in the effects of baicalin [10]. Shieh et al. reported that baicalin-induced apoptosis is Bcl-2-dependent instead of p53-dependent in human leukaemic cells [11]. Huang et al. reported that baicalin-induced apoptosis may reduce telomerase activity in HL-60 leukaemic cells [12]. The combination of baicalin and other agents has shown good efficacy in human leukaemic cells [13]. Inhibition of the PI3K/Akt signalling pathway was considered as a mechanism involved in leukaemic cell apoptosis [14]. It has been reported that baicalin induced apoptosis in CA46 lymphoma cells via down-regulation of the PI3K/Akt signalling pathway [7]. Our previous work also suggested that baicalin-induced apoptosis is related to down-regulation of the PI3K/Akt signalling pathway in HL-60/ADM cells [15]. Therefore, baicalin is considered as a good candidate for combination therapy against leukaemia.

It has been well documented that overexpression of drug resistance-related genes, like multidrug resistance related protein 1 (MDR1), multidrug resistance protein 1 (MRP1), lung resistance-related protein (LRP) and breast cancer-related protein (BCRP) strongly extrudes chemotherapeutic agents crossing the cellular membrane and reduces the intracellular bioavailability and concentration of drugs, leading to MDR [16]. In this regard, downregulation of the expression of these genes may be an important strategy against MDR in leukaemia. Recent studies have shown that down-regulating the expression of the MDR1 gene by osthol [17], or MDR1, MRP1 and BCRP genes by homeobox B4 knockdown (HOXB4) [16] share the same mechanisms, that is, inhibition of the PI3K/Akt signalling pathway can reverse resistance to ADM in myelogenous K562/ADM leukaemic cells. HL-60 is another leukaemic cell line widely used in MDR research. The expression of the LRP gene in HL-60 cells remains a subject of controversy. In 1995, Solvak et al. reported high expression of the LRP and MRP genes in HL-60 cells. Interestedly, another study reported that LRP gene expression was low in HL-60 cells, although highly expressed in relapsed patients with MDR [18]. Thus, it is hypothesised that regular HL-60 cells and HL-60 cells with MDR may share different biological characteristics.

Based on this evidence, we selected an ADM-resistant human leukaemic cell line, namely HL-60/ADM cells for the subsequent experiments. We assessed the expression of MRP and LRP genes in these resistant cells and investigated the synergistic effect of baicalin with ADM, namely the reversal of resistance to ADM. Additionally, we investigated the mechanisms involved in this process by observing changes in MDR-related gene expression and the PI3K/ Akt signalling pathway induced by baicalin administration. 


\section{Cellular Physiology Cell Physiol Biochem 2017;43:419-430

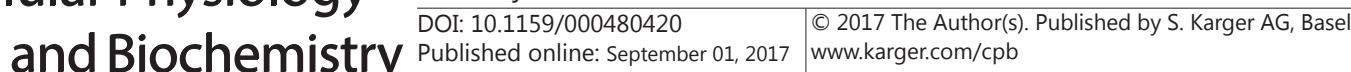 \\ Zheng et al.: Synergistic Effect of Baicalin and Adriamycin in HL-60/ADM Cells}

\section{Materials and Methods}

\section{Cell lines}

The regular acute myeloid leukaemia (AML) HL-60 cell line along with ADM-resistant cell line, namely HL-60/ADM cells were obtained from the Chinese Academy of Medical Sciences, Tianjin Institute of Haematology. HL-60 ADM cells were cultured in RPMI1640 medium (Gibco, NY, USA) containing $0.4 \mu \mathrm{g} / \mathrm{L}$ $\mathrm{ADM}$; and 10\% foetal bovine serum (Hao Yang, China) to keep the ADM-resistance Cells were incubated at $37^{\circ} \mathrm{C}$ under saturated humidity and were passaged once every 2 days. Two weeks prior to the experiment, the culture medium was replaced with non-ADM RPMI1640 medium in HL-60/ADM cells. Cells from the logarithmic growth phase were used for the experiments.

\section{Chemicals and reagents}

Sterile baicalin was purchased from a pharmaceutical company (Qingze, China). Baicalin purity was 99.0\%, determined through high-performance liquid chromatography. The drug was dissolved in dimethyl sulfoxide (DMSO) (Sigma, USA) and stored at $-20^{\circ} \mathrm{C}$. ADM was obtained from Sigma. The following primary antibodies were used in the analysis: anti-Akt, anti-p-Akt and LY294002 were purchased from Cell Signaling Technology, Inc. (Danvers, USA); MRP1 antibody was purchased from Becton Dickinson (BD) Bioscience Corporation (Franklin Lakes, USA); LRP antibody was obtained from Santa Cruz Biotechnology (Santa Cruz, USA); Bcl-2 antibody was purchased from eBioscience Corporation (San Diego, USA) and $\beta$-actin mouse monoclonal antibody was purchased from NeoMarkers, Inc. (Fremont, USA).

\section{Experimental approach to confirm the effects of baicalin on HL-60/ADM cells}

MTT assays. Cytotoxicity assays: Cytotoxicity assays were used to determine the viability and proliferation of HL-60 and HL-60/ADM cells after administration of ADM. HL-60 and HL-60/ADM cells from the logarithmic growth phase were seeded into 96-well plates at a density of $2.0 \times 10^{5}$ per well. The ADM dilution was subsequently added. The final concentrations of ADM in wells containing HL-60 cells were $0,0.05,0.1$, $0.2,0.4$ and $0.8 \mu \mathrm{mol} / \mathrm{L}$, and those in wells containing HL-60/ADM cells were 0, 5, 10, 20, 40 and $80 \mu \mathrm{mol} / \mathrm{L}$. Each concentration was performed in triplicate. The cells were cultured in a humidified $5 \% \mathrm{CO}_{2}$ incubator at $37^{\circ} \mathrm{C}$ for $48 \mathrm{~h}$, and the quantity of viable cells was determined. The resistance index was calculated as the half maximal inhibitory concentration (IC50) of ADM-resistant cells/IC50 of sensitive cells.

Inhibition of HL-60/ADM cell proliferation by low doses of baicalin in combination with ADM: Based on the inhibition rate of baicalin in HL60/ADM cell proliferation (Fig. 1), baicalin solutions $(10 \mu \mathrm{mol} / \mathrm{L})$ were selected to reverse drug resistance. ADM-resistant cells were seeded in 96-well plates, and 5, 10, 20, 40 or 80 $\mu \mathrm{mol} / \mathrm{L}$ ADM solutions were added, followed by $10 \mu \mathrm{mol} / \mathrm{L}$ baicalin. Controls included those containing cells in the absence of the study drugs and vice versa. Plates were incubated at $37^{\circ} \mathrm{C}$. After $48 \mathrm{~h}$, the optical density (OD) was measured. The IC50 of baicalin in combination with ADM was calculated using the OD values.

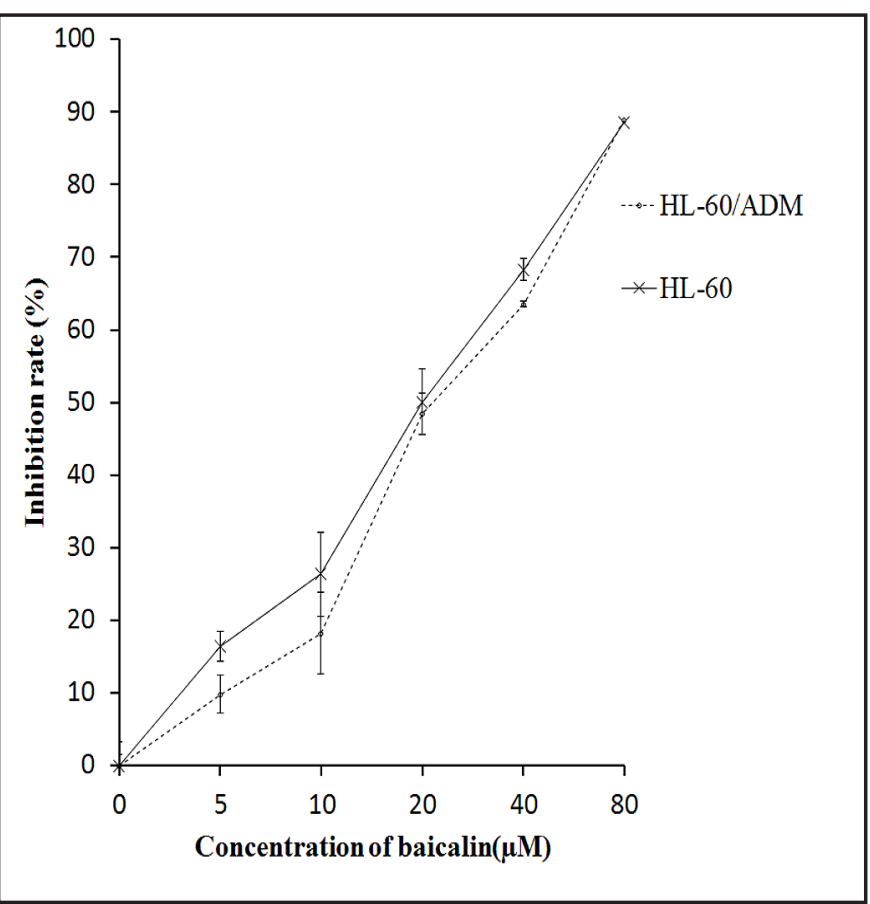

Fig. 1. Dose-response curve of administration of baicalin. The doses of 5 and $10 \mu \mathrm{M}$ exhibited weak cytotoxicity (inhibition rate $\leq 20 \%$ ). We, therefore, selected these as the administration doses for the experiments. 


\section{Cellular Physiology Cell Physiol Biochem 2017;43:419-430 \begin{tabular}{l|l|l}
\hline and $10.1159 / 000480420$ & $\begin{array}{l}\text { C) } 2017 \text { The Author(s). Published by S. Karger AG, Basel } \\
\text { www.karger.com/cpb }\end{array}$
\end{tabular} \\ Zheng et al.: Synergistic Effect of Baicalin and Adriamycin in HL-60/ADM Cells}

The reversal fold (RF) was calculated as the IC50 without reversal agents/IC50 with reversal agents. Doses (5 and $10 \mu \mathrm{M}$ ), which exhibited weak cytotoxicity (inhibition rate $\leq 20 \%$, Fig. 1), were selected as administration doses for the following experiments.

Flow cytometry assays. Intracellular drug accumulation assay: Flow cytometry was used to determine the ADM fluorescent-positive ratio in HL-60/ADM cells, since ADM is autofluorescent. Cells were cultured in a 6-well plate for $24 \mathrm{~h}$ and subsequently incubated with $10 \mu \mathrm{mol} / \mathrm{L}$ baicalin. HL-60 and HL-60/ADM cells not treated with baicalin were used as controls. The cells were incubated for $24 \mathrm{~h}$. ADM was added to a final concentration of $10 \mu \mathrm{mol} / \mathrm{L}$ and the cells were incubated for another $2 \mathrm{~h}$. All the cells were collected and washed twice. The fluorescent-positive rate in HL-60/ADM cells was analysed using a BD FACSCanto II cytometer (BD Biosciences, USA). The intracellular median fluorescence intensity (MFI) associated with ADM was detected via fluorescence-activated cell sorting. The excitation and emission wavelengths were 488 and $550 \mathrm{~nm}$, respectively. A filter was used to determine red fluorescence intensity.

Annexin V apoptosis assay: The cells were treated with DMSO alone or with various concentrations of baicalin for $12 \mathrm{~h}$ to determine the induction of apoptosis by baicalin in HL-60/ADM cells. The cells were subsequently washed with ice-cold phosphate-buffered saline and incubated with annexin V-FITC (BD Pharmigen, USA) and propidium iodide (Trevigen, USA). The rate of apoptosis was analysed by flow cytometry.

\section{Investigation of the mechanisms related to the effects of baicalin}

Real-time polymerase chain reaction (PCR) analysis to measure the expression of MRP1, LRP and Bcl2 genes. HL-60/ADM cells were pretreated with different concentrations of baicalin or RPMI1640 for 24 $\mathrm{h}$ to measure the mRNA expression of MRP1, LRP and Bcl-2 in HL-60 cells and HL-60/ADM cells, along with changes induced by baicalin administration. Total mRNA was extracted and reverse transcribed. The transcription levels of MRP1, LRP and Bcl-2 [19] were detected by quantitative real-time PCR using the iCycler real-time detection system (Bio-Rad, USA) in a two-step method. The hot start enzyme was activated at $95^{\circ} \mathrm{C}$ for $5 \mathrm{~min}$. Subsequently, cDNA was amplified for 40 cycles, which consisted of denaturation at $95^{\circ} \mathrm{C}$ for $15 \mathrm{~s}$ and annealing/extension at $58^{\circ} \mathrm{C}$ for $30 \mathrm{~s}$. A melt curve analysis was then performed (the temperature was $55^{\circ} \mathrm{C}$ for $1 \mathrm{~min}$ and then increased by $0.5^{\circ} \mathrm{C}$ every $10 \mathrm{~s}$ ) to detect the formation of primer-derived trimmers and dimmers. The primer sequences used were the following: MRP1 forward, 5'-GGTCACGCACAGCATG-3', and reverse, 5'-GTACACGGAAAGCTTGAC-3'; LRP forward, 5'-AGTCAGAAGCCG AGAAAG-3', and reverse, 5'-CCCAGCCACAGCAAGGT-3'; Bcl-2 forward, 5'-CGACGACTTCTCCCGCCGCTACCGC-3', and reverse, 5 '-CCGCATGCTGGG GCCGTACAGTTCC-3' and $\beta$-actin forward, 5'-GTGGGCGCCCCAGACACCA- 3 ', and reverse, 5'-CTCCTTAATGTCACGCACGATTC-3'. $\beta$-actin was used as internal control.

\section{Western blotting analysis}

Western blotting analysis was used to analyse changes in the expression of proteins related to the study treatment. Following different treatments, HL-60/ADM cells were harvested and lysed. Protein concentrations were measured using the $\mathrm{DC}^{\mathrm{TM}}$ protein assay kit (Bio-Rad, USA). Electrophoresis was performed in $8-12 \%$ sodium dodecyl sulphate polyacrylamide gel, and samples were subsequently transferred onto a polyvinylidene fluoride membrane. Western blotting analysis was conducted in accordance with the kit instructions. The following primary antibodies were used for the analysis: Poly (ADP-ribose) polymerase (PARP), caspase-3, MRP1, LRP1, Bcl-2, Akt, p-Akt, LY294002 and $\beta$-actin. $\beta$-actin was used as the internal reference. Finally, an enhanced chemiluminescence detection system (Pierce, USA) was used for exposure. Quantitative analyses of protein expression were performed using an Image-ProPlus system (Media Cybernetics, USA).

\section{Statistical analysis}

Data were recorded as the mean \pm standard deviation and analysed using SPSS 19.0.0 (IBM, USA). Differences between the groups were analysed using two-way analysis of variance followed by Bonferroni post hoc correction for multiple comparisons. All tests were two-sided. $p<0.05$ was set as significant difference. 


\section{$\begin{array}{ll}\text { Cellular Physiology } & \text { Cell Physiol Biochem 2017;43:419-430 } \\ \text { DOI: 10.1159/000480420 } & 0 \text { 2017 The Author(s). Published by S. Karger AG, Basel }\end{array}$ \begin{tabular}{ll|l} 
DOI: 10.1159/000480420 & $\begin{array}{l}\text { O } 2017 \text { The Author(s). Published by S. Karger AG, Basel } \\
\text { www.karger.com/cpb }\end{array}$ \\
\cline { 2 - 4 }
\end{tabular} \\ Zheng et al.: Synergistic Effect of Baicalin and Adriamycin in HL-60/ADM Cells}

\section{Results}

In the present study, we confirmed the cytotoxic effects of baicalin in HL-60/ADM cells and subsequently investigated the mechanisms involved in the therapeutic effects of baicalin.

Confirmation of the effects of baicalin in $H L-60 / A D M$ cells

Reversal of resistance to ADM by baicalin in HL-60/ADM cells. HL-60/ADM exhibited strong resistance to ADM (246.6-fold increase, Fig. 2A), with IC50 of $30.58 \mu \mathrm{M}$ vs 0.12 $\mu \mathrm{M}$ in HL-60/ADM and HL-60 cells, respectively. However, the combination of baicalin with ADM showed significant reduction in the IC50 of ADM in HL-60/ADM cells. The high concentration of baicalin $(10 \mu \mathrm{M})$ exhibited the stronger effect (3.02 vs 1.89 , Fig. 2B). This finding was reproduced in different concentrations of ADM (Fig. 2C). These data confirmed that administration of baicalin may have a synergistic effect with ADM, reversing drug resistance in HL-60/ADM cells.

Baicalin-induced apoptosis in HL-60/ADM cells by increasing the intracellular accumulation of ADM. The results of Annexin V staining indicated that baicalin induced apoptosis in HL$60 / \mathrm{ADM}$ cells, and administration of $10 \mu \mathrm{M}$ baicalin exhibited a stronger effect than that of 5 $\mu \mathrm{M}$ (Fig. 3A). These findings were also confirmed by Western blotting analysis (Fig. 3B). The

Fig. 2. Verification of the toxic effects of baicalin in HL-60/ADM cells. A. IC50 values of $\mathrm{ADM}$ in different cells. HL-60/ADM cells exhibited approximately 246.6-fold increase in resistance to ADM compared with regular HL-60 cells. B. IC50 values of ADM in different HL-60/ADM cells. Baicalin significantly reduced resistance compared with administration of ADM alone. C. Growth curves of HL-60/ADM cells treated by $\mathrm{ADM}$ alone, $\mathrm{ADM}+5 \mu \mathrm{M}$ baicalin and ADM $+10 \mu \mathrm{M}$ baicalin. Baicalin exhibited inhibitory effects on HL$60 /$ ADM cells. IC50 $=$ half maximal inhibitory concentration, $\mathrm{RF}=$ reversal fold, $\mathrm{ADM}=$ Adriamycin, $*$ means $\mathrm{p}<0.05$.

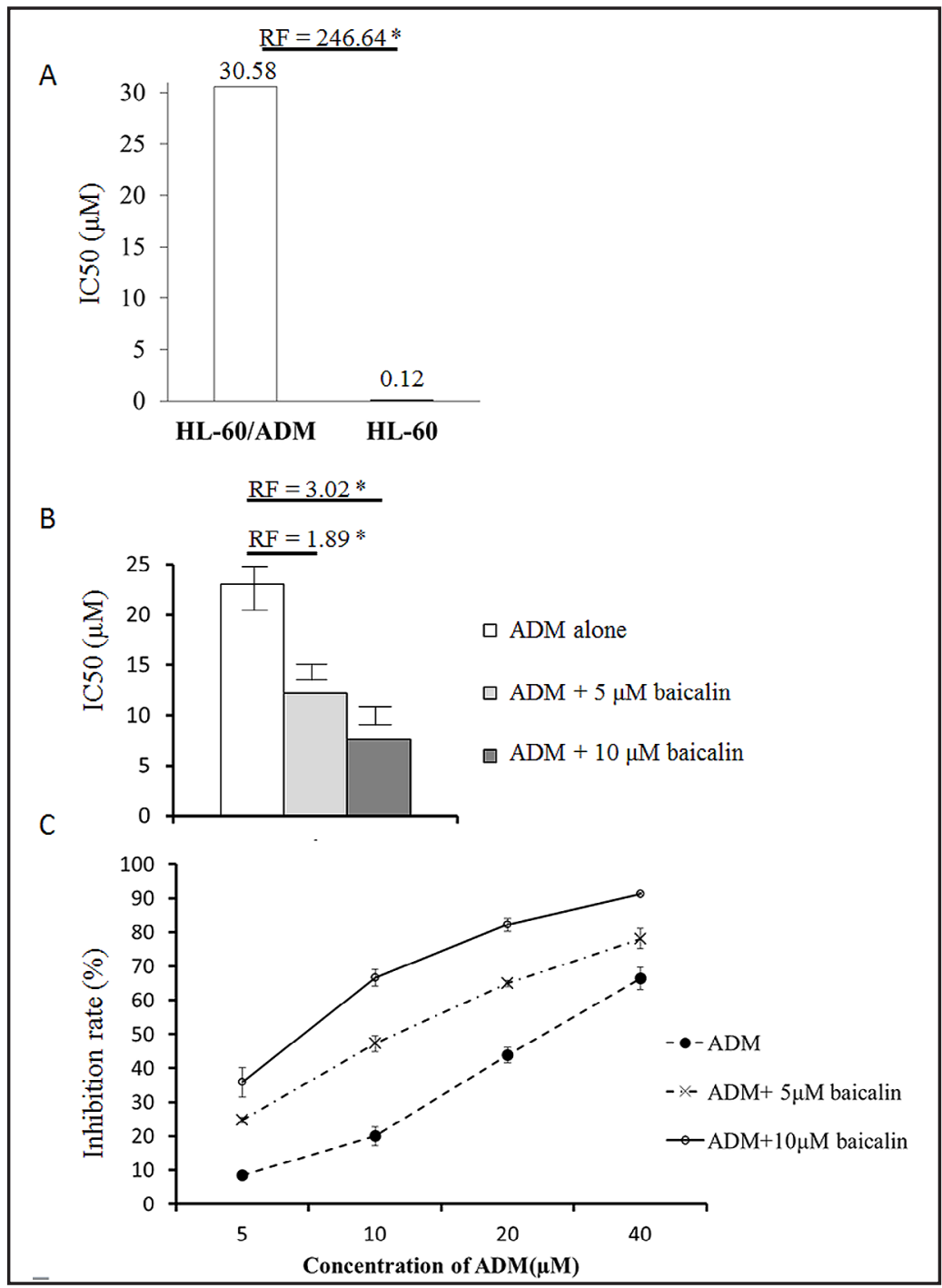




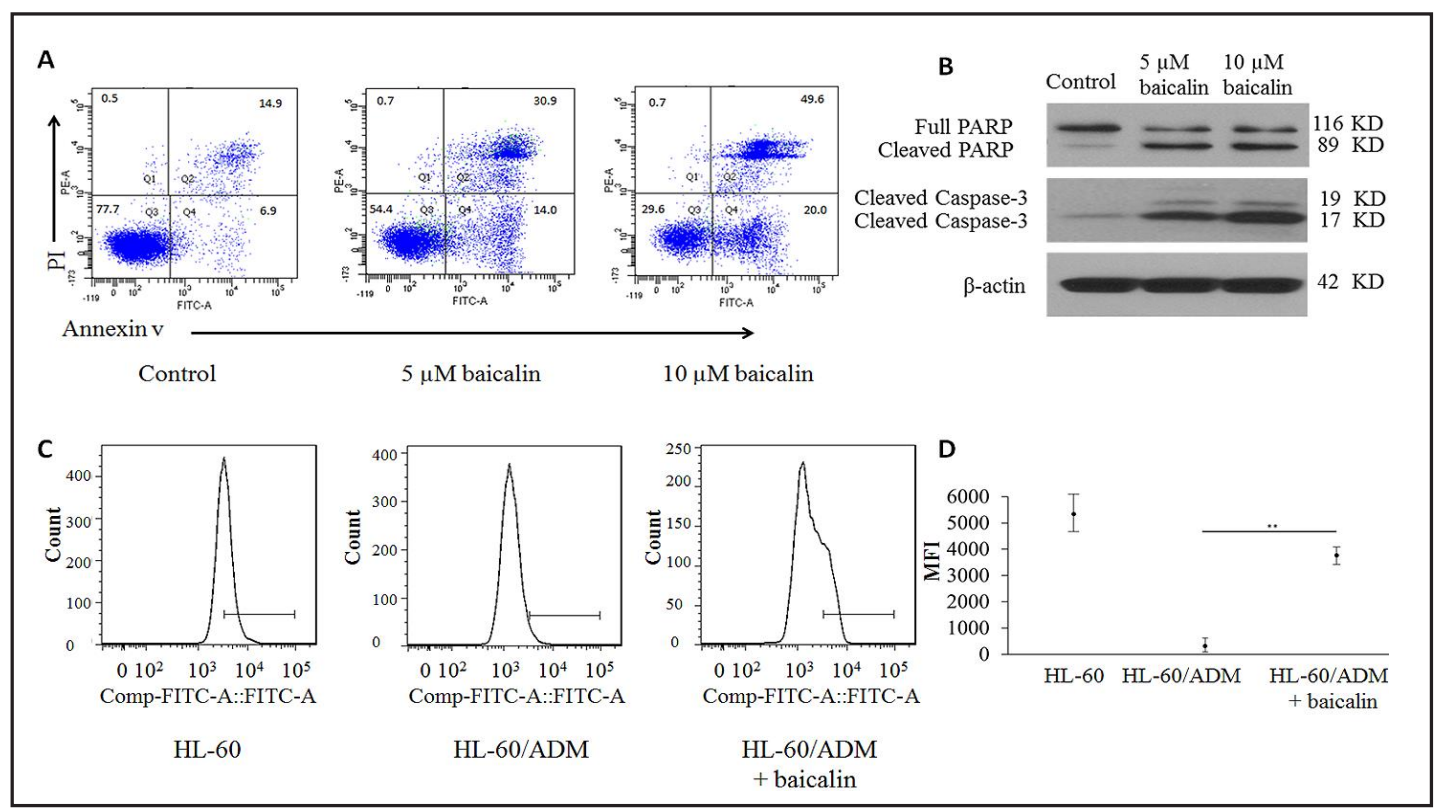

Fig. 3. Increase in the intracellular accumulation of ADM by baicalin may trigger apoptosis in HL-60/ADM cells. A. Results of Annexin V staining verified that baicalin induced apoptosis in HL-60/ADM cells. The dose of $10 \mu \mathrm{M}$ exhibited better efficacy than that of $5 \mu \mathrm{M}$. B. Images of Western blotting analysis for apoptosisrelated proteins also confirmed the efficacy of baicalin. The dose of $10 \mu \mathrm{M}$ exhibited the strongest efficacy. These results showed that baicalin induces apoptosis in HL-60/ADM cells. C. The fluorescence characteristics of ADM in different cells. Administration of baicalin significantly improved the intracellular accumulation of ADM in HL-60/ADM cells. D. Intracellular MFI value in different cells. Administration of baicalin significantly enhanced the intracellular MFI value in HL-60/ADM cells, indicating that the intracellular accumulation of ADM was increased. MFI = median fluorescence intensity, PI = propidium iodide, $* *$ means $\mathrm{p}<0.01$..

results of the quantitative analysis are shown in Fig. 4 (Fig. 4). Full PARP was down-regulated due to consumption during the processes of apoptosis, whereas apoptosisrelated proteins (cleaved PARP 89 KD and cleaved caspase-3 17, 19 KD) were up-regulated by baicalin. This is further evidence that baicalin induces apoptosis in HL60/ADM cells.

Interestingly, we found that baicalin increased the intracellular concentration of ADM

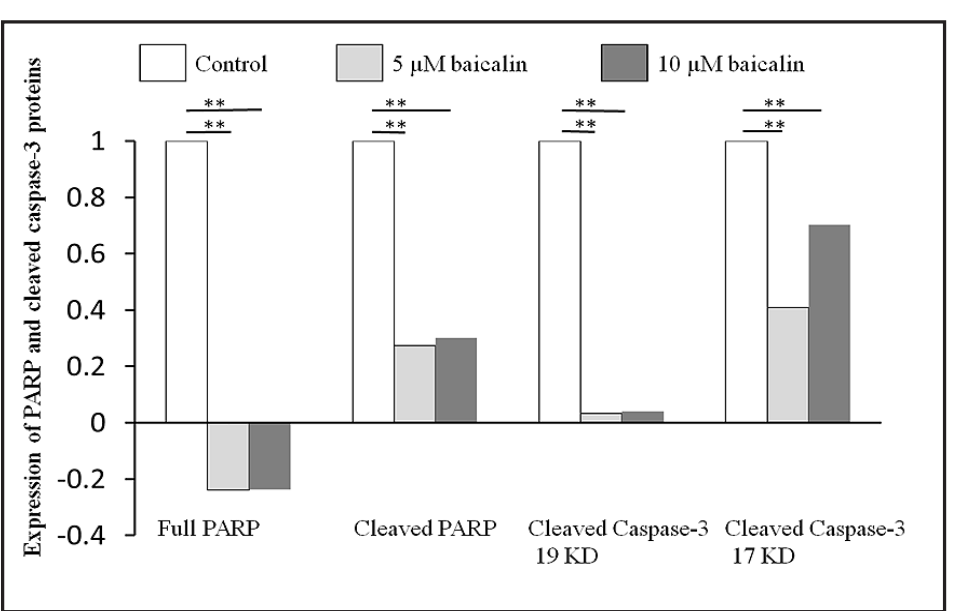

Fig. 4. Quantitative analysis of expression of PARP and cleaved caspase-3 proteinsThe dose of $10 \mu \mathrm{M}$ exhibited stronger cytotoxicity, as shown in Fig. 3B.

when administered in combination with ADM. Fig. 3C is a representative image obtained by flow cytometry. The spectra of the HL-60/ADM + baicalin group exhibited a higher level of intracellular ADM (Fig. 3C). The MFI values of HL-60 and HL-60/ADM cells were 5337.33 \pm 


\section{$\begin{array}{lll}\text { Cellular Physiology } & \text { Cell Physiol Biochem 2017;43:419-430 } \\ \text { DOI: 10.1159/000480420 } & 0 \text { 2017 The Author(s). Published by S. Karger AG, Basel }\end{array}$

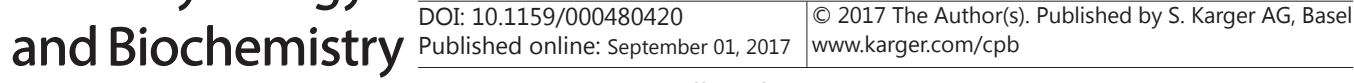 \\ Zheng et al.: Synergistic Effect of Baicalin and Adriamycin in HL-60/ADM Cells}

705.60 and $320.33 \pm 69.90$, respectively. Following treatment with $10 \mu \mathrm{mol} / \mathrm{L}$ baicalin for 48 $\mathrm{h}$, the intracellular accumulation of ADM increased to $3765.67 \pm 402.79$, namely 6.98-fold increase compared with the negative control in HL-60/ADM cells $(p<0.01)$ (Fig. 3D).

These findings indicated that baicalin induced apoptosis in HL-60/ADM cells, in which the increase in intracellular accumulation of ADM may play a role.

\section{Investigations of the mechanisms related to the effects of baicalin}

Confirmation of the expression of MDR-related genes and Bcl-2 gene in HL-60 and HL60/ADM cells. MRP1, LRP and Bcl-2 genes were expressed in both HL-60 and HL-60/ADM cells. Moreover, the expression of these genes was significantly higher in HL-60/ADM cells (Fig. 5A and 5B). We confirmed that the biological characteristics of HL-60/ADM cells were different from those of HL-60. HL-60/ADM cells were therefore employed for subsequent studies.

Down-regulation of the expression of MDR-related genes and Bcl-2 gene in HL-60/ADM

Fig. 5. Baicalin downregulated the expression of the MDR-related genes in HL-60/ADM cells. A. Quantitative analysis of mRNA expression of MRP1, LRP and Bcl-2 in HL-60 and HL-60/ADM cells. The mRNA levels of MRP1, LRP and Bcl-2 in HL-60/ADM cells were significantly higher than those in HL-60 cells; B. Western blotting analysis of MRP1, LRP and Bcl-2 in HL-60 and HL-60/ADM cells; C. Quantitative analysis of mRNA expression of MRP1, LRP and Bcl-2 affected by the administration of baicalin. The mRNA levels of MRP1, LRP and Bcl-2 in HL-60/ ADM cells were significantly reduced by the administration of baicalin (5 and $10 \mu \mathrm{M}$ ); D. Western blotting analysis of MRP1, LRP and Bcl- 2 affected by the administration of baicalin. E. Western blotting analysis of MRP1 and LRP affected by the admin-
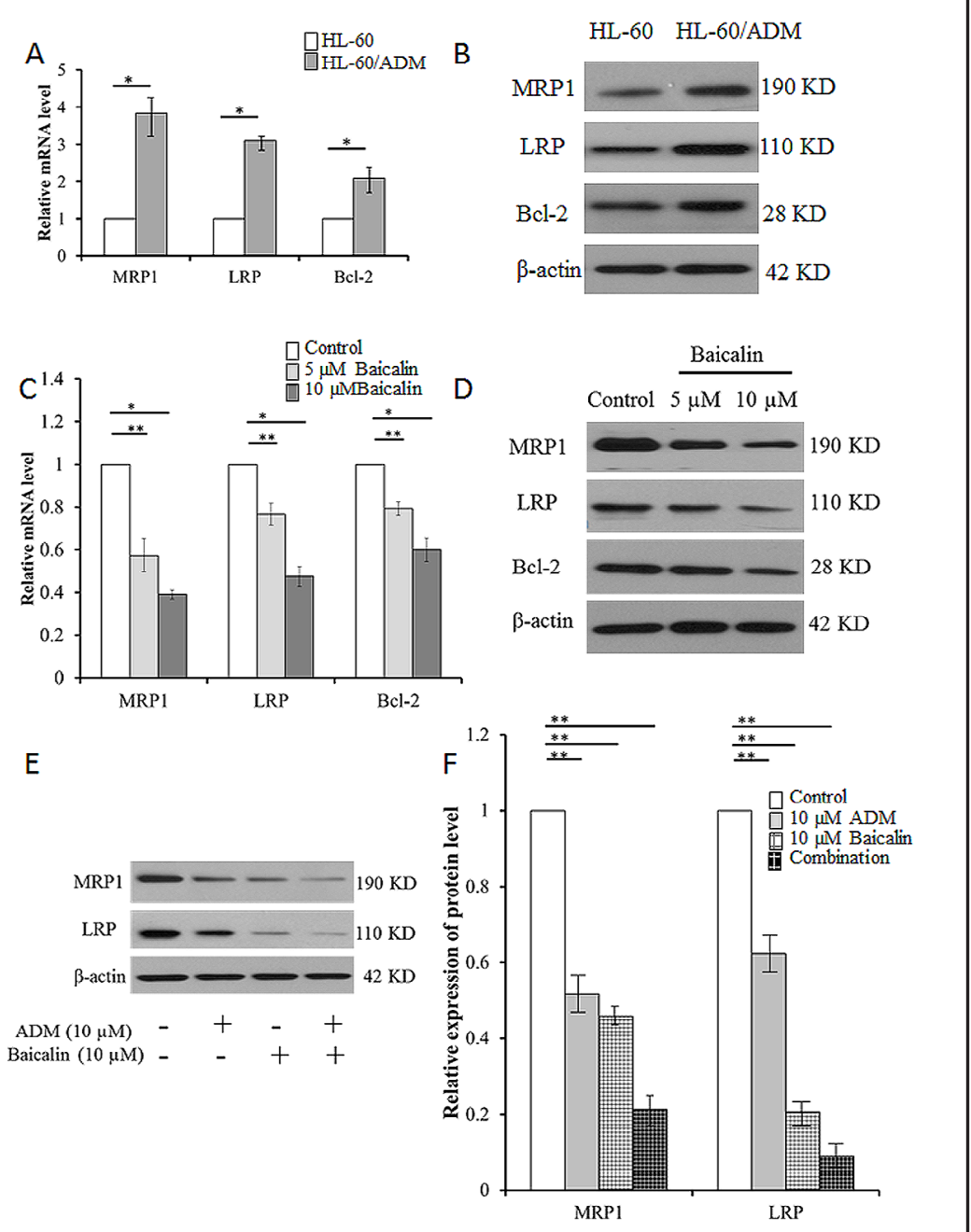

istration of baicalin and

ADM; F. Quantitative analysis of the protein expression of MRP1 and LRP affected by the administration of baicalin and ADM. Expression of MRP1 and LRP in HL-60/ADM cells was significantly decreased by the administration of both baicalin and ADM. Moreover, the combination achieved greater efficacy. MRP1 = multidrug resistance-associated protein $1, \mathrm{LRP}=$ lung-resistance protein, Bcl-2 = B-cell lymphoma 2 , ** means $\mathrm{p}<0.01$. 
A

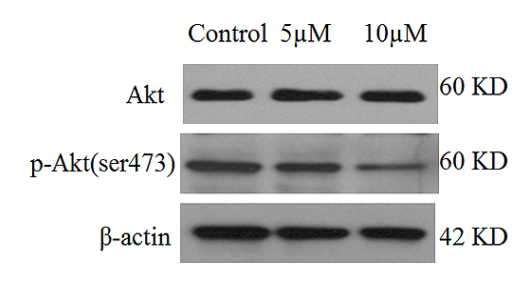

C

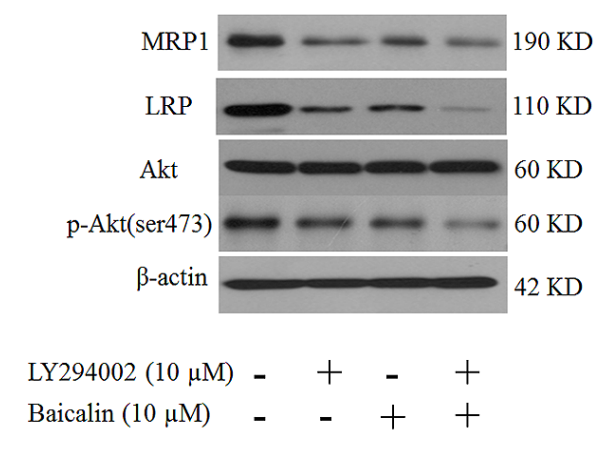

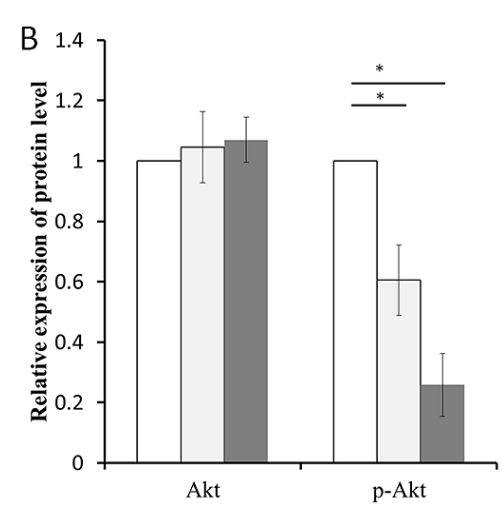

D

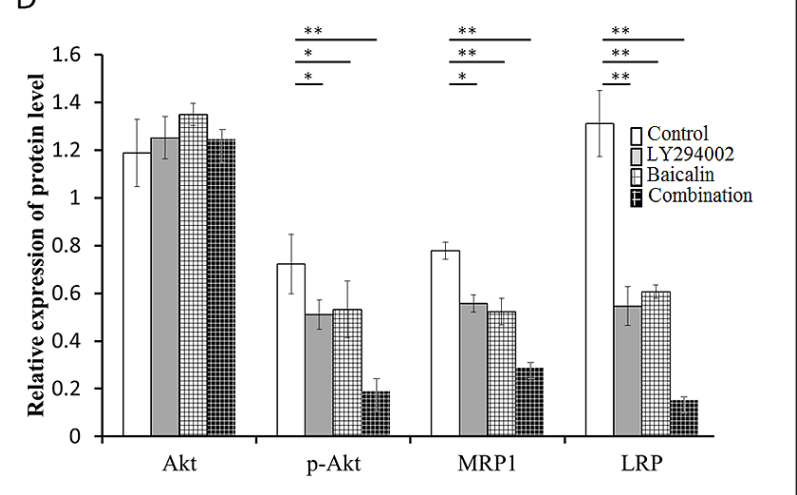

Fig. 6. Effects of baicalin on the PI3K/Akt signalling pathway. A. Expression of Akt and p-Akt affected by the administration of baicalin; B. Quantitative analysis of the expression of Akt and p-Akt. We did not find any significant difference in Akt expression among the groups. However, the administration of baicalin (5 and $10 \mu \mathrm{M})$ significantly down-regulated the expression of p-Akt. C. Expression of MRP1, LRP, Akt and p-Akt affected by the administration of LY294002 and baicalin; D. Quantitative analysis of the expression of MRP1, LRP, Akt and p-Akt. No changes were seen in Akt expression among groups. Expression of p-Akt, MRP1 and LRP were significantly down-regulated by LY294002 and baicalin. Moreover, the combination of LY294002 and baicalin achieved a stronger inhibition, indicating a synergistic effect of LY294002 and baicalin. * means $\mathrm{p}<0.05,{ }^{* *}$ means $\mathrm{p}<0.01$.

cells by baicalin. The expression of MRP1, LRP and Bcl-2 genes in HL-60/ADM cells treated by baicalin was investigated. The results were analogous in these genes. Baicalin significantly down-regulated the expression of these genes in HL-60/ADM cells. The larger dose $(10 \mu \mathrm{M})$ exhibited a stronger down-regulation than that of the lower dose (5 $\mu \mathrm{M})$ (Fig. 5C and 5D).

The effect of the down-regulation of MRP1 and LRP in HL-60/ADM cells treated by ADM, baicalin and their combination was also compared. Administration of ADM or baicalin significantly decreased gene expression. However, the combination of ADM and baicalin exerted the strongest down-regulation effect, indicating a synergistic effect of ADM and baicalin (Fig. 5E and 5F).

Inhibition of the PI3K/Akt signalling pathway may play a role in the mechanisms of baicalin-reversed MDR. With regard to the expression of Akt/p-Akt in HL-60/ADM cells, we found that administration of baicalin significantly down-regulated the expression of p-Akt, and the larger dose $(10 \mu \mathrm{M})$ exhibited a stronger down-regulation effect than that of the lower dose $(5 \mu \mathrm{M})$. However, the expression of Akt was not affected by baicalin (Fig. 6A and 6B).

The inhibitory effects of baicalin with LY294002 on PI3K/Akt signalling pathway were also investigated. Interestingly, the administration of LY294002 yielded similar results as baicalin. The expression of p-Akt, MRP1 and LRP was significantly down-regulated by KARGER 


\section{Cellular Physiology Cell Physiol Biochem 2017;43:419-430 and Biochemistry \begin{tabular}{l|l} 
DOI: $10.1159 / 000480420$ & $\begin{array}{l}\text { (c) } 2017 \text { The Author(s). Published by S. Karger AG, Basel } \\
\text { www.karger.com/cpb }\end{array}$
\end{tabular} \\ Zheng et al.: Synergistic Effect of Baicalin and Adriamycin in HL-60/ADM Cells}

both LY294002 and baicalin. Moreover, the combination of LY294002 and baicalin exerted a stronger down-regulation effect, indicating a synergistic effect of LY294002 and baicalin. Akt was unaffected by LY294002, baicalin and their combined administration (Fig. 6C and 6D).

These findings indicated that inhibition of the PI3K/Akt signalling pathway may play a role in the mechanisms of baicalin-reversed MDR.

\section{Discussion}

In the present study, we employed an ADM-resistant leukaemic cell line using HL60/ADM cells. The expression of MRP1, LRP and Bcl-2 genes in the resistant HL-60/ADM cells was confirmed. Using these HL$60 /$ ADM cells, we found that baicalin exhibited a synergistic effect with ADM by increasing

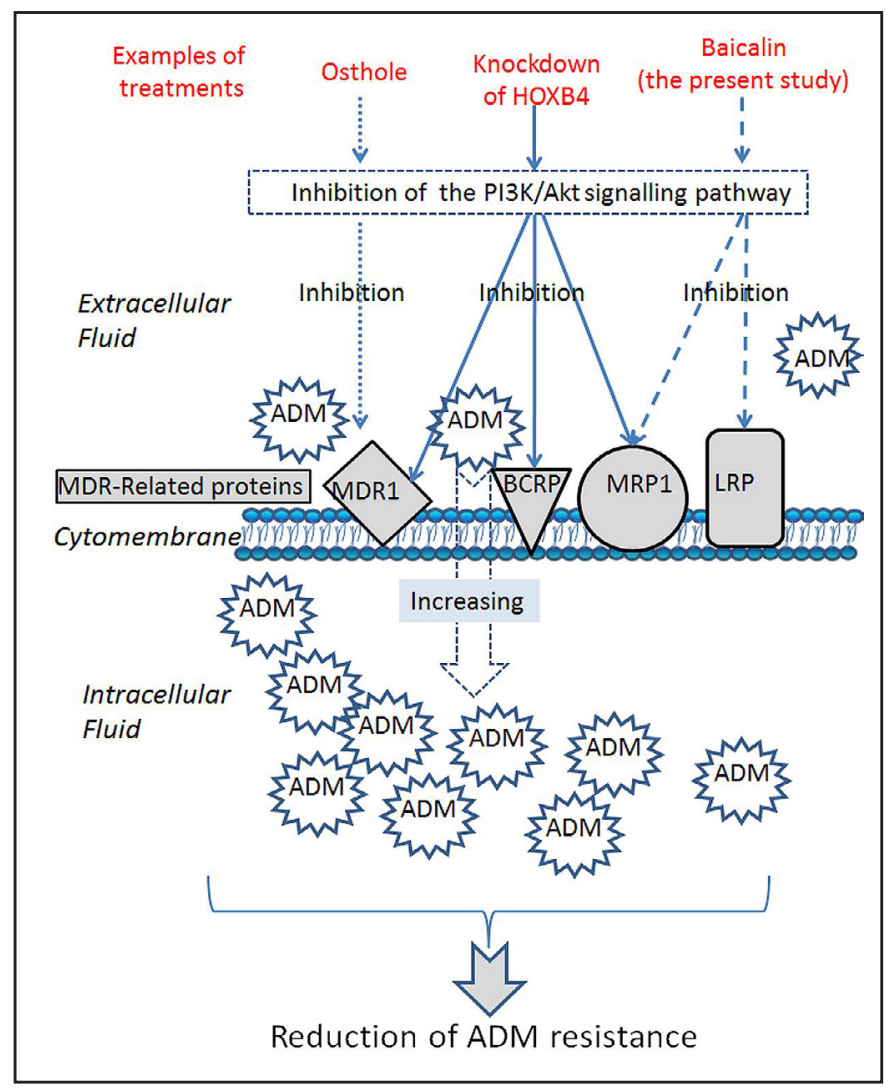

Fig. 7. Potential mechanisms of combination treatments reversing resistance to ADM. the intracellular accumulation of ADM, reducing resistance to ADM. We found that MDR-related genes, namely MRP1, LRP gene and an apoptosis gene, namely Bcl-2 were highly expressed in HL-60/ADM cells, whereas they were down-regulated by baicalin. The combination of ADM and baicalin resulted in the strongest down-regulation, confirming the synergistic effect of ADM and baicalin. Moreover, it was shown that both MDR-related genes and p-Akt are down-regulated by administration of baicalin and LY294002, a specific antagonist of the PI3K/Akt signalling pathway. Baicalin and LY294002 exhibited a synergistic effect. These findings suggest that inhibition of the PI3K/Akt signalling pathway may play a role in the down-regulation of MDR-related genes, consequently reversing ADM resistance state in leukaemic cells. Interestingly, we firstly confirmed that the expression of LRP was higher in ADM-resistant HL-60 cells, and subsequently provided evidence of the synergistic effect of baicalin and ADM using these HL-60/ADM cells. These findings indicated that HL-60/ADM cells are a more appropriate cell line model for the investigation of MDR than the regular HL-60 cells. Baicalin may be a potential candidate for combination chemotherapy with ADM. Clinical validation of these findings is warranted.

Drug resistance in $H L-60 / A D M$ cells. Inspired by the results of a previous study on the dispersed expression of the LRP gene among regular HL-60 cells and relapsed AML patients [18], we selected ADM-resistant leukaemic HL-60 cells for the following studies. The significant difference in IC50 between HL-60/ADM and HL-60 indicated that HL-60/ADM cells are highly resistant to ADM. Compared with K562/ADM cells [17], the HL-60/ADM cells exhibited higher ADM-resistance (RF: 246.6 vs 38.55). In addition, the IC50 of HL-60/ADM cells was shown to be affected by the administration of baicalin (Fig. 2).

The expression of MDR-related genes and Bcl-2 gene was also investigated. In our preliminary experiments, we found high expression of MRP1, LRP and Bcl-2 genes, unlike 


\section{Cellular Physiology Cell Physiol Biochem 2017;43:419-430 \begin{tabular}{ll|l} 
and Biochemistry Published online: September 01, 2017 & $\begin{array}{l}\text { (c) } 2017 \text { The Author(s). Published by S. Karger AG, Basel } \\
\text { www.karger.com/cpb }\end{array}$ \\
\hline
\end{tabular} \\ Zheng et al.: Synergistic Effect of Baicalin and Adriamycin in HL-60/ADM Cells}

the MDR1 and BCRP genes, in accordance with previous results on HL-60 cells [20]. The expression of MRP1, LRP and Bcl-2 genes in HL-60/ADM cells was significantly higher than that observed in HL-60 cells (Fig. 5 A and 5B).

These data suggested that in comparison with regular HL-60 cells, HL-60/ADM cells are a satisfactory and sensitive representation of ADM-resistant cells. These cells are suitable and recommended for the investigation of MDR.

The synergistic effect of baicalin and ADM. Administration of baicalin enhanced the inhibition and apoptosis rate in HL-60/ADM cells $(10 \mu \mathrm{M}>5 \mu \mathrm{M})$, and the synergistic effect of baicalin and ADM was confirmed. We also confirmed that administration of baicalin significantly enhanced the intracellular levels of ADM. Therefore, it was deduced that baicalin reduces resistance to ADM by increasing the intracellular accumulation of ADM in HL-60/ADM cells. Our results are in accordance with previous studies $[16,17]$ (Fig. 3). It has been reported that administration of osthol [17] or suppressing the expression of the HOXB4 gene [16] increases the intracellular accumulation of ADM in K562/ADM cells. The consistent results from studies using different treatments and different ADM-resistant cell lines suggested that increasing the intracellular levels of ADM is a common mechanism of combination therapy with ADM, reversing resistance to ADM.

The roles of MDR-related genes and PI3K/Akt signalling pathway. The roles of MDRrelated genes have been well documented in previous studies $[9,16,17,20]$. However, the genes involved vary between studies, most likely due to differences in treatment. Wang et al. indicated that HOXB4 gene knockdown affects the expression of MDR1, MRP1 and BCRP genes [16], whereas administration of osthol only affects MDR1 [17]. The use of different leukaemic cell line models may also account for the varied gene expression observed between studies. The present study showed that in HL-60/ADM cells, MRP1 and LRP were the only MDR-related genes expressed. Verification of these findings using other cell lines is indispensable in future studies to develop an overall understanding of the MDR-related genes involved in this process. In the present study, administration of baicalin induced significant down-regulation of the expression of the MRP1 and LRP genes $(10 \mu \mathrm{M}>5 \mu \mathrm{M}$, Fig. 5C and 5D). Additionally, the combination of ADM and baicalin enhanced the down-regulation of MRP1 and LRP, exhibiting another synergistic effect of this combination (Fig. 5E and 5F). In accordance with Wang's studies $[16,17]$, down-regulating the expression of MDR-related genes is considered as another common mechanism improving the crossing of the cellular membrane by chemotherapeutic agents. The intracellular bioavailability and concentration of the drug was subsequently increased, resulting in enhanced apoptosis in leukaemic cells.

Our data showed similar changes in two different kinds of proteins, namely MDR-related MRP1 and LRP, and PI3K/Akt signalling pathway-related p-Akt. The expression of these proteins can be down-regulated by baicalin and specific PI3K/Akt antagonists. Baicalin and LY294002 exhibited a synergistic effect (Fig. 6). These data strongly suggest that inhibition of the PI3K/Akt signalling pathway induced by baicalin may play a role in the down-regulation of the expression of MDR-related genes. The PI3K/Akt pathway is an important molecular mechanism underlying MDR, as shown in previous studies [16, 17, 21].

Collectively, the findings of the present study together with those from previous studies using ADM-resistant cells lines (K562/ADM) [16, 17], demonstrate that the combination of ADM with other agents may share the same mechanism (Fig. 7). Combination drugs (such as baicalin and osthol) or treatments (such as knockdown of the HOXB4 gene) suppress the PI3K/Akt signalling pathway, triggering the down-regulation of MDR-related gene expression. The overexpressing state of MDR-related genes is corrected. The crossing of the cellular membrane by chemotherapeutic agents is consequently changed and the intracellular bioavailability and concentration of the drugs are enhanced. Moreover, resistance to agents such as ADM is reversed and the state of MDR is ameliorated. These mechanisms should be verified in other drug-resistant leukaemic cell lines. Moreover, this study proposes the use of agents in combination with ADM for the treatment of AML. Such compound ought to be sensitive to the PI3K/Akt signalling pathway and to other leukaemic cell lines, while being less toxic. 


\section{Cellular Physiology Cell Physiol Biochem 2017;43:419-430 \begin{tabular}{ll|l} 
and BiOChemistry & DOI: 10.1159/000480420 & $\begin{array}{l}\text { (C) } 2017 \text { The Author(s). Published by S. Karger AG, Basel } \\
\text { www.karger.com/cpb }\end{array}$
\end{tabular}

\section{Conclusion}

We verified the expression of MDR-related genes in HL-60/ADM cells and found that MRP1, LRP and Bcl-2 were expressed in both HL-60 and HL-60/ADM cells. HL-60/ADM cells exhibited higher expression. HL-60/ADM cells are more sensitive and appropriate for the investigation of MDR than regular HL-60 cells. The findings of the present study provided evidence of the synergistic effect of baicalin and ADM in HL-60/ADM cells. Baicalin may be considered as a potential candidate against resistant leukaemia, although further study is warranted to verify these findings. Combination treatments with ADM may share the same mechanism: suppression of the PI3K/Akt signalling pathway, down-regulation of the MDRrelated genes, enhancement of the intracellular levels of ADM and consequent amelioration of the MDR state in leukaemic cells.

\section{Acknowledgements}

This study was supported by the grant from National Nature and Science Foundation, China (No.81370630. 2014-2014) and The National Key Research and Development Program (2016YFC0902800). The authors would like to thank Chinese Academy of Medical Sciences, Tianjin Institute of Haematology for providing the HL-60 and HL-60/ADM cell lines.

The authors would like to thank Enago (www.enago.jp) for the English language review.

\section{Disclosure Statement}

The authors declare that there are no conflicts of interest.

\section{References}

1 Wang H, Zhang Y, Bai R, Wang M, Du S: Baicalin attenuates alcoholic liver injury through modulation of hepatic oxidative stress, inflammation and sonic hedgehog pathway in rats. Cell Physiol Biochem 2016;39:1129-1140.

-2 Xi Y, Wu M, Li H, Dong S, Luo E, Gu M, Shen X, Jiang Y, Liu Y, Liu H: Baicalin attenuates high fat diet-induced obesity and liver dysfunction: dose-response and potential role of CaMKK $\beta /$ AMPK/ACC pathway. Cell Physiol Biochem 2015;35:2349-2359.

3 Wang J, Masika J, Zhou J, Wang J, Zhu M, Luo H, Hu X, Zhang L, Tang M, Gao L: Traditional Chinese medicine baicalin suppresses mESCs proliferation through inhibition of miR-294 expression. Cell Physiol Biochem 2015;35:1868-1876.

4 Ikezoe T, Chen SS, Heber D, Taguchi H, Koeffler HP: Baicalin is a major component of PC - SPES which inhibits the proliferation of human cancer cells via apoptosis and cell cycle arrest. Prostate 2001;49:285292.

5 Zhang Y, Liao P, Li W, Hu D, Chen L, Guan S: Baicalin Attenuates Cardiac Dysfunction and Myocardial Remodeling in a Chronic Pressure-Overload Mice Model. Cell Physiol Biochem 2017;41:849-864.

6 Chan FL, Choi H, Chen Z, Chan PS, Huang Y: Induction of apoptosis in prostate cancer cell lines by a flavonoid, baicalin. Cancer Lett 2000;160:219-228.

-7 Huang Y, Hu J, Zheng J, Li J, Wei T, Zheng Z, Chen Y: Down-regulation of the PI3K/Akt signaling pathway and induction of apoptosis in CA46 Burkitt lymphoma cells by baicalin. J Exp Clin Cancer Res 2012;31:48.

-8 Zhang H-B, Lu P, Guo Q-Y, Zhang Z-H, Meng X-Y: Baicalein induces apoptosis in esophageal squamous cell carcinoma cells through modulation of the PI3K/Akt pathway. Oncol Lett 2013;5:722-728.

-9 Lu H-F, Hsueh S-C, Ho Y-T, Kao M-C, Yang J-S, Chiu T-H, Huamg S-Y, Lin C-C, Chung J-G: ROS mediates baicalin-induced apoptosis in human promyelocytic leukemia HL-60 cells through the expression of the Gadd153 and mitochondrial-dependent pathway. Anticancer Res 2007;27:117-125.

10 Li Y, Tyan Y, Kuo H, Chang W, Hsia T, Chung J: Baicalein induced in vitro apoptosis undergo caspases activity in human promyelocytic leukemia HL-60 cells. Food Chem Toxicol 2004;42:37-43. 


\section{Cellular Physiology Cell Physiol Biochem 2017;43:419-430 \begin{tabular}{l|l|l} 
DOI: 10.1159/000480420 & $\begin{array}{l}\text { O 2017 The Author(s). Published by S. Karger AG, Basel } \\
\text { www.karger.com/cpb }\end{array}$
\end{tabular} \\ Zheng et al.: Synergistic Effect of Baicalin and Adriamycin in HL-60/ADM Cells}

11 Shieh D-E, Cheng H-Y, Yen M-H, Chiang L-C, Lin C-C: Baicalin-induced apoptosis is mediated by Bcl-2dependent, but not p53-dependent, pathway in human leukemia cell lines. Am J Chin Med 2006;34:245261.

12 Huang S-T, Wang C-Y, Yang R-C, Chu C-J, Wu H-T, Pang J-HS: Wogonin, an active compound in Scutellaria baicalensis, induces apoptosis and reduces telomerase activity in the HL-60 leukemia cells. Phytomedicine 2010;17:47-54.

13 Ren X, Zhang Y, Li C, Wang H, Jiang Z, Zhang Z, Guo Q, Song G, Bi K, Jiang G: Enhancement of baicalin by hexamethylene bisacetamide on the induction of apoptosis contributes to simultaneous activation of the intrinsic and extrinsic apoptotic pathways in human leukemia cells. Oncol Rep 2013;30:2071-2080.

14 Chen Y, Li J, Hu J, Zheng J, Zheng Z, Liu T, Lin Z, Lin M: Emodin enhances ATRA-induced differentiation and induces apoptosis in acute myeloid leukemia cells. Int J Oncol 2014;45:2076-2084.

15 Zheng J, Hu J-D, Chen Y-Y, Chen B-Y, Huang Y, Zheng Z-H, Liu T-B: Baicalin induces apoptosis in leukemia HL-60/ADR cells via possible down-regulation of the PI3K/Akt signaling pathway. Asian Pac J Cancer Prev 2012;13:1119-1124.

16 Wang H, Jia X-H, Chen J-R, Yi Y-J, Wang J-Y, Li Y-J, Xie S-Y: HOXB4 knockdown reverses multidrug resistance of human myelogenous leukemia K562/ADM cells by downregulating P-gp, MRP1 and BCRP expression via PI3K/Akt signaling pathway. Int J Oncol 2016;49:2529-2537.

17 Wang H, Jia X-H, Chen J-R, Wang J-Y, Li Y-J: Osthole shows the potential to overcome P-glycoprotein-mediated multidrug resistance in human myelogenous leukemia K562/ADM cells by inhibiting the PI3K/Akt signaling pathway. Oncol Rep 2016;35:3659-3668.

18 Gil L, Styczynski J, Dytfeld D, Debski R, Kazmierczak M, Kolodziej B, Rafinska B, Kubicka M, Nowicki A, Komarnicki M: Activity of bortezomib in adult de novo and relapsed acute myeloid leukemia. Anticancer Res 2007;27:4021-4025.

19 Liu Y, Su L, Lin Q, Han Y, You P, Fan Q: Induction of c-mip by IL-17 plays an important role in adriamycininduced podocyte damage. Cell Physiol Biochem 2015;36:1274-1290.

20 Slovak ML, Ho JP, Cole SP, Deeley RG, Greenberger L, de Vries EG, Broxterman HJ, Scheffer GL, Scheper RJ: The LRP gene encoding a major vault protein associated with drug resistance maps proximal to MRP on chromosome 16: evidence that chromosome breakage plays a key role in MRP or LRP gene amplification. Cancer Res 1995;55:4214-4219.

21 Zhang H-T, Wang W-W, Ren L-H, Zhao X-X, Wang Z-H, Zhuang D-L, Bai Y-N: The mTORC2/Akt/NFkB Pathway-Mediated Activation of TRPC6 Participates in Adriamycin-Induced Podocyte Apoptosis. Cell Physiol Biochem 2016;40:1079-1093. 